\title{
Perceptions of Hospital-Dependent Patients on Their Needs for Hospitalization
}

\author{
Tao Liu, MD* ${ }^{1 *}$ Eliza Kiwak, BA¹, Mary E. Tinetti, MD¹
}

${ }^{1}$ Section of Geriatrics, Department of Internal Medicine, Yale University School of Medicine, New Haven, Connecticut.

\begin{abstract}
In the United States, older adults account for a significant proportion of hospitalizations, and a subset become hospital-dependent, for reasons that are unclear. We conducted a qualitative study to explore these individuals' perspectives on their need for hospitalizations. Twenty patients hospitalized at an academic medical center underwent semistructured qualitative interviews. Criteria for selection included age 65 and older, at least three hospitalizations over six months, admission to the medical service at the time of the study, did not meet criteria for chronic critical illness, was not comfort measures only, and did not have a conservator.
\end{abstract}

Interviews were audiotaped, transcribed, and inductively analyzed. The major themes derived were the necessity and inevitability of hospitalizations ("You have to bring me in here"), feeling safe in the hospital ("It makes me feel more secure"), patients hospitalized despite having outside medical and social support ("I have everything"), and inadequate goalsof-care discussions ("It just doesn't occur to me"). Results suggested that candid discussions about health trajectories are needed to ensure hospitalization is consistent with the patient's realistic health priorities. Journal of Hospital Medicine 2017;12:450-453. (c) 2017 Society of Hospital Medicine
In the United States, patients 65 years old or older accounted for more than one third of inpatient stays and $42 \%$ of inpatient care spending in $2012 .{ }^{1}$ Despite the identification of risk factors, the implementation of an array of interventions, and the institution of penalties on hospitals, a subset of older adults continues to spend significant time in the hospital. ${ }^{2,3}$

Hospital dependency is a concept that was only recently described. It identifies patients who improve while in the hospital but quickly deteriorate after leaving the hospital, resulting in recurring hospitalizations. ${ }^{4}$ Although little is known about hospital-dependent patients, studies have explored patients' perspectives on readmissions. ${ }^{5,6}$ Nevertheless, it remains unclear whether there are individuals for whom frequent and prolonged hospitalizations are appropriate, and whether there are undisclosed factors that, if addressed, could decrease their hospital dependency. We conducted an exploratory study to ascertain hospital-dependent patients' perspectives on their needs for hospitalizations.

\section{METHODS}

\section{Study Design}

This study was approved by the Yale University Institutional Review Board. From March 2015 to September 2015, Dr.

\footnotetext{
*Address for correspondence and reprint requests: Tao Liu, MD, Department of Internal Medicine-Geriatrics, Yale School of Medicine, 333 Cedar Street, PO Box 208025, New Haven, CT 06520-8025; Telephone: 412-273-5914; Fax: 203-688-4209; E-mail: taowuhan@gmail.com

Additional Supporting Information may be found in the online version of this article.

Received: August 29, 2016; Revised: November 17, 2016; Accepted: December 2, 2016
}

2017 Society of Hospital Medicine DOI 10.12788/jhm.2756
Liu conducted semistructured explorative interviews with patients on the medical units of an academic medical center. Dr. Liu was not directly involved in the care of these patients. An interview guide that includes open-ended questions was created to elicit patients' perspectives on their need for hospitalizations, health status, and outside-hospital support. This guide was pilot-tested with 6 patients, whose transcripts were not included in the final analysis, to assess for ease of understanding. After the pilot interviews, the questions were revised, and the final guide consists of 12 questions (Supplemental Table).

\section{Recruitment}

We used predetermined criteria and a purposeful sampling strategy to select potential study participants. We identified participants by periodically ( once a week) reviewing the electronic medical records of all patients admitted to the medicine service during the study period. Eligible patients were 65 years old or older and had at least 3 hospitalizations over the preceding 6 months. Patients were excluded if they met our chronic critical illness criteria: mechanical ventilation for more than 21 days, history of tracheotomy for failed weaning from mechanical ventilation, ${ }^{7}$ presence of a conservator, or admission only for comfort measures. Participants were recruited until no new themes emerged.

\section{Data Collection}

Twenty-nine patients were eligible. We obtained permission from their inpatient providers to approach them about the study. Of the 29 patients, 26 agreed to be interviewed, and 3 declined. Of the 26 participants, 6 underwent pilot interviews, and 20 underwent formal interviews with use of the finalized interview guide. The interviews, conducted in the hospital while the participants were hospitalized, lasted 17 minutes on average. The interviews were transcribed and iteratively 
analyzed. The themes that emerged from the initial interviews were further explored and validated in subsequent interviews. Interviews were conducted until theoretical saturation was reached and no new themes were derived from them. Demographic information, including age, sex, ethnicity, and marital status, was also collected.

\section{Analysis}

Interviews were digitally recorded and transcribed. Independently, two investigators used Atlas Ti software to analyze and code the interview transcriptions. An inductive approach was used to identify new codes from the data. ${ }^{8}$ The coders then met to discuss repeating ideas based on the codes. When a code was identified by one coder but not the other, or when there was disagreement about interpretation of a code, the coders returned to the relevant text to reach consensus and to determine whether to include or discard the code. ${ }^{9}$ We then organized and reorganized repeating ideas based on their conceptual similarities to determine the themes and subthemes. ${ }^{9}$

\section{RESULTS}

Twenty patients participated in the formal interviews. Participants' baseline characteristics are listed in Table 1, and four dominant themes, and their subthemes and exemplary quotations, are listed in Table 2.

\section{Perspectives on Hospital Care}

Participants perceived their hospitalizations as inevitable and necessary for survival: "I think if I haven't come to the hospital, I probably would have died." Furthermore, participants thought only the hospital had the resources to help them ("The medications they were giving me ... you can get that in the hospital but not outside the hospital") and sustain them ("You are like an old car, and it breaks down little by little, so you have to go in periodically and get the problem fixed, so you will drive it around for a while").

Feeling Safe in Hospital. Asked how being in the hospital makes them feel, participants attributed their feelings of safety to the constant observation, the availability of providers and nurses, and the idea that hospital care is helping. As one participant stated, "Makes me feel safer in case you go into something like cardiac arrest. You are right here where they can help you."

Outside-Hospital Support. Despite multiple hospitalizations, most participants reported having social support ("I have the aide. I got the nurses come in. I have my daughter ..."), physical support, and medical support ("I have all the doctors") outside the hospital. A minority of participants questioned the usefulness of the services. One participant described declining the help of visiting nurses because she wanted to be independent and thought that, despite recurrent hospitalizations for physical symptoms, she still had the ability to manage her own medications.

Goals-of-Care Discussion. Some participants reported inadequate discussions about goals of care, health priorities, and health trajectories. In their reports, this inadequacy included
TABLE 1. Characteristics of Participants $(n=20)$

\begin{tabular}{|c|c|}
\hline Characteristic & $n(\%)^{a}$ \\
\hline \multicolumn{2}{|l|}{ Age, y } \\
\hline Mean (SD) & $81(6)$ \\
\hline $65-74$ & $2(10)$ \\
\hline $75-84$ & $10(50)$ \\
\hline$\geq 85$ & $8(40)$ \\
\hline Women & $12(60)$ \\
\hline \multicolumn{2}{|l|}{ Race/ethnicity } \\
\hline White & $17(85)$ \\
\hline Black & $3(15)$ \\
\hline \multicolumn{2}{|l|}{ Marital status } \\
\hline Married & $10(50)$ \\
\hline Widowed & $9(45)$ \\
\hline Single & $1(5)$ \\
\hline Mean (SD) medications, $\mathrm{n}$ & $16(6)$ \\
\hline \multicolumn{2}{|l|}{ Residence before admission } \\
\hline Home & $16(80)$ \\
\hline Skilled nursing facility & $3(15)$ \\
\hline Assisted living facility & $1(5)$ \\
\hline \multicolumn{2}{|l|}{ Location after hospital discharge } \\
\hline Home & $12(60)$ \\
\hline Skilled nursing facility & $8(40)$ \\
\hline Mean (SD) length of stay per admission, d & $7.1(6)$ \\
\hline Mean (SD) hospitalizations in past 12 months, n & $6(4)$ \\
\hline \multicolumn{2}{|l|}{ Principal diagnosis for current admission } \\
\hline COPD/asthma exacerbation & $5(25)$ \\
\hline CHF exacerbation & $4(20)$ \\
\hline Urinary traction infection & $3(15)$ \\
\hline Pneumonia & $2(10)$ \\
\hline Other & $6(30)$ \\
\hline \multicolumn{2}{|l|}{ Chronic condition } \\
\hline CAD/CHF/cardiomyopathy & $14(70)$ \\
\hline Atrial fibrillation & $10(50)$ \\
\hline Hypertension & $15(75)$ \\
\hline Diabetes & $13(65)$ \\
\hline Chronic kidney disease & $11(55)$ \\
\hline COPD & $9(45)$ \\
\hline Depression & $5(25)$ \\
\hline Stroke/transient ischemic attack & $3(15)$ \\
\hline Cancer except nonmelanoma skin cancers & $3(15)$ \\
\hline
\end{tabular}

${ }^{a}$ Except where indicated otherwise.

NOTE: Abbreviations: CAD, coronary artery disease; CHF, congestive heart failure; $\mathrm{COPD}$, chronic obstructive pulmonary disease; SD: standard deviation.

not thinking about their goals, despite continued health decline. One participant stated, “Oh, God, I don't know if I had any conversation like that. ... I think until it is really brought to the front, you don't make a decision really if you don't have to." Citing the value of a more established relationship and deeper trust, participants preferred having these serious and personal discussions with their ambulatory care clinicians: "Because I know my doctor much closer. I have been with him for a number of years. The doctors in the hospital seem to be nice and competent, but I don't know them." 
TABLE 2. Older Adults' Perspectives on Their Need for Hospitalizations

\begin{tabular}{ll}
\hline Subthemes & Theme 1. Perspectives about hospital care \\
\hline Subtheme 1. & Participants express their hospital care needs and their reasons for seeking hospital care and for experiencing multiple hospitalizations \\
Need for hospital care & "I think if I haven't come to the hospital, I probably would have died"—A1, 85-year-old Caucasian man \\
& "If I break down and can't breathe, then you have to bring me in here, and they got to do all these stuff when I come in to the hospital"—A3, 86-year-old African \\
& American woman
\end{tabular}

Subtheme 2.

Why hospital care

"When I am sick at home, I feel like I don't want to go to the hospital, but if I have to go to the hospital I have to go because I want to get over this feeling ... so I know I have no choice ... they give me something over there to fix me up a little"—A17, 85-year-old Caucasian man

"The medications they were giving me to see if they were working, you can get that in the hospital but not outside the hospital—-that's why my doctors outside tell me to get into the emergency room"-A15, 78-year-old Caucasian man

"They have all things in the hospital that I don't have at home, you know, so I can't take no $x$-ray at home, I have to come into the hospital and do it, you know—all the doctors come in here to see me, they can't come in my house, so they see me in hospital"—A3, 86-year-old African American woman

Subtheme 3.

"Well, it gets better when I come to the hospital ... say I go home and stay about a month, and the same thing happens to me again, and then I come back to the

Why multiple hospitalizations hospital again and they put me on the medication again. And then it holds me up for about a month again, then I would be back in"-A3, 86-year-old African American woman

"Well, I have a lot of chronic problems, they are probably related to my age, like the heart and the kidney ... and so you are like an old car, and it breaks down little by little, so you have to go in periodically and get the problem fixed, so you will drive it around for a while and something else would break down, and you go and get it fixed"-A8, 85-year-old Caucasian woman

\begin{tabular}{ll}
\hline & Theme 2. Feeling safe in hospital \\
No Subthemes & Participants describe their feelings about hospital safety
\end{tabular}

"Just being around them makes you feel safer, makes me feel safer in case you go into something like cardiac arrest. You are right here where they can help you"A18, 75-year-old African American woman

"It makes me more secure knowing that there are people who can help cure me"—A6, 84-year-old Caucasian man

"It makes me feel more secure. Well, I feel that I am in good hands, that they can take care of me and do things that I can't do"—A7, 78-year-old Caucasian woman

Theme 3. Recurrent hospitalizations despite having support outside hospital
Participants describe their perspectives on their out-of-hospital experiences-the social and
physical support they may or may not receive, their medical care, and why they decline home support

Subtheme 1. physical support they may or may not receive, their medical care, and why they decline home support

Home social and physical

"My youngest daughter carries me through this whole thing ... she knows all the medications, all the illnesses, all the doctors. She makes all my appointments, she support does all that stuff. She goes with me to all the appointments"-A15, 78-year-old Caucasian man

"I have everything. I have the aide. I got the nurses come in. I have my daughter and my daughter-in-law"—A4, 89-year-old Caucasian woman

Subtheme 2.

Ambulatory medical care

"I have a heart doctor, I have an eye doctor, I have a chest doctor whatever you call it, I have all the doctors"—A3, 86-year-old African American woman

"I have a nurse who comes usually at least once a week ... I will continue to see her until I am well enough that I won't need her ... I have seen her many times, so she understands my case"-A7, 78-year-old Caucasian woman

"I have a good doctor and if one is not available for whatever reason, there is always someone taking over for him. I have been with the same doctor for 25 years, and I have had the same cardiologist for about 20 years, so that makes a difference, and both are very caring"-A8, 85-year-old Caucasian woman

Subtheme 3.

Feels home care is declining

or services are not helping

"They told me that I could have the visiting nurse come in and put my pills out because I take about 16 pills a day. And I refuse. I said I know just where everything is ... and I said the visiting nurse would just upset me because they don't know where things are" —A9, 89-year-old Caucasian woman

"I saw one, and that was the only one that came in every other day that wrap my leg. And to me, that was ridiculous because, as I said, they didn't wrap it the way it should have been wrapped anyway. So you get discouraged, so why are you doing this. All you are doing is giving government money"-A12, 80-year-old Caucasian woman

\begin{tabular}{ll}
\hline & Theme 4. Goals-of-care discussions \\
& Participants express reasons for not having conversations about goals of care and \\
& identify the clinicians with whom they would prefer to have such conversations \\
Subthemes & "Can I tell why I don't want to talk to them, because usually it just doesn't occur to me"—A11, 87-year-old Caucasian man \\
\hline Subtheme 1. & "I noticed that our intern has been hitting my wife with those questions a little bit, hasn't been particularly at me directly. Usually we have too much other stuff to worry \\
Reasons for not having & about"—A16, 77-year-old Caucasian man \\
healthcare goals & "Oh, God, I don't know if I had any conversation like that. I don't know, I think until it is really brought to the front, you don't make a decision really if you don't have \\
& to"-A19, 75-year-old Caucasian woman \\
\hline Subtheme 2. & "Because I know my doctor much closer. I have been with him for a number of years. The doctors in the hospital seem to be nice and competent, but I don't know \\
With whom to have & them"-A1, 85-year-old Caucasian man \\
conversations about & "Well, I have a primary doctor. He knows mostly about me, and that's who I would talk to ... because you don't have any relationship with [providers in the hospital]. When \\
healthcare goals & you see [a primary care provider] for 20 years, you have a relationship"—A7, 78-year-old Caucasian woman \\
& "Yeah, my primary doctor, I don't do nothing without consulting him first. I trust [providers in the hospital], don't get me wrong, but I will tell my primary doctor things \\
that I won't tell the new ones" -A4, 89-year-old Caucasian woman
\end{tabular}

\section{DISCUSSION}

Participants considered their hospitalizations a necessity and reported feeling safe in the hospital. Given that most already had support outside the hospital, increasing community services may be inadequate to alter participants' perceived hospital care needs. On the other hand, a few participants reported declining services that might have prevented hospitaliza- tions. Although there has been a study of treatment refusal among older adults with advanced illnesses, ${ }^{10}$ not much is known about refusal of services among this population. Investigators should examine the reasons for refusing services and the effect that refusal has on hospitalizations. Furthermore, although it would have been informative to ascertain clinician perspectives as well, we focused on patient perspec- 
tives because less is known on this topic.

Some participants noted their lack of discussion with their clinicians about healthcare goals and probable health trajectories. Barriers to goals-of-care discussion among this highly vulnerable population have been researched from the perspectives of clinicians and other health professionals but not patients themselves. ${ }^{11,12}$ Of particular concern in our study is the participant-noted lack of discussion about health trajectories and health priorities, given the decline that occurs in this population and even in those with good care. This inadequacy in discussion suggests continued hospital care may not always be consistent with a patient's goals. Patients' desire to have this discussion with their clinicians, with whom they have a relationship, supports the need to involve ambulatory care clinicians, or ensure these patients are cared for by the same clinicians, across healthcare settings. ${ }^{13,14}$ Whoever provides the care, the clinician must align treatment with the patient's goal, whether it is to continue hospital-level care or to transition to palliative care. Such an approach also reflects the core elements of person-centered care. ${ }^{15}$

\section{Study Limitations}

Participants were recruited from the medicine service at a single large academic center, limiting the study's generalizability to patients admitted to surgical services or community hospitals. The patients in this small sample were English-speaking and predominantly Caucasian, so our findings may not represent the perspectives of non-English-speaking or minority patients. We did not perform statistical analysis to quantify intercoder reliability. Last, as this was a qualitative study, we cannot comment on the relative importance or prevalence of the reasons cited for frequent hospitalizations, and we cannot estimate the proportion of patients who had recurrent hospitalizations and were hospital-dependent.

\section{Implication}

Although quantitative research is needed to confirm our findings, the hospital-dependent patients in this study thought their survival required hospital-level care and resources. From their perspective, increasing posthospital and community support may be insufficient to prevent some hospitalizations. The lack of goals-of-care discussion supports attempts to increase efforts to facilitate discussion about health trajectories and health priorities between patients and their preferred clinicians.

\section{Acknowledgments}

The authors thank Dr. Grace Jenq for providing feedback on the study design.

Disclosure: Nothing to report.

\section{References}

1. Weiss AJ, Elixhauser A. Overview of Hospital Stays in the United States, 2012: Statistical Brief 180. Rockville, MD: Agency for Healthcare Research and Quality, Healthcare Cost and Utilization Project; 2014. http://www.ncbi.nlm.nih.gov/books/ NBK259100/. Published October 2014. Accessed February 17, 2016.

2. Auerbach AD, Kripalani S, Vasilevskis EE, et al. Preventability and causes of readmissions in a national cohort of general medicine patients. JAMA Intern Med. 2016;176(4):484-493

3. Donzé JD, Williams MV, Robinson EJ, et al. International validity of the HOSPITAL score to predict 30-day potentially avoidable hospital readmissions. JAMA Intern Med. 2016;176(4):496-502.

4. Reuben DB, Tinetti ME. The hospital-dependent patient. $N$ Engl J Med. 2014;370(8):694-697.

5. Enguidanos S, Coulourides Kogan AM, Schreibeis-Baum H, Lendon J, Lorenz K. "Because I was sick": seriously ill veterans' perspectives on reason for 30-day readmissions. J Am Geriatr Soc. 2015;63(3):537-542.

6. Kangovi S, Grande D, Meehan P, Mitra N, Shannon R, Long JA. Perceptions of readmitted patients on the transition from hospital to home. J Hosp Med. 2012;7(9):709-712

7. Lamas D. Chronic critical illness. N Engl J Med. 2014;370(2):175-177.

8. Saldana J. Fundamentals of Qualitative Research. Cary, NC: Oxford University Press; 2011.

9. Auerbach CF, Silverstein LB. Qualitative Data: An Introduction to Coding and Analysis. New York, NY: New York University Press; 2003.

10. Rothman MD, Van Ness PH, O'Leary JR, Fried TR. Refusal of medical and surgical interventions by older persons with advanced chronic disease. J Gen Intern Med. 2007;22(7):982-987.

11. You JJ, Downar J, Fowler RA, et al; Canadian Researchers at the End of Life Network. Barriers to goals of care discussions with seriously ill hospitalized patients and their families: a multicenter survey of clinicians. JAMA Intern Med. 2015;175(4):549-556

12. Schoenborn NL, Bowman TL 2nd, Cayea D, Pollack CE, Feeser S, Boyd C. Primary care practitioners' views on incorporating long-term prognosis in the care of older adults. JAMA Intern Med. 2016;176(5):671-678.

13. Arora VM, Prochaska ML, Farnan JM, et al. Problems after discharge and understanding of communication with their primary care physicians among hospitalized seniors: a mixed methods study. J Hosp Med. 2010;5(7):385-391.

14. Jones CD, Vu MB, O'Donnell CM, et al. A failure to communicate: a qualitative exploration of care coordination between hospitalists and primary care providers around patient hospitalizations. J Gen Intern Med. 2015;30(4):417-424.

15. American Geriatrics Society Expert Panel on Person-Centered Care. Person-centered care: a definition and essential elements. J Am Geriatr Soc. 2016;64(1):15-18. 